\title{
Conservación in vitro: una perspectiva para el manejo de los recursos fitogenéticos
}

\section{In-vitro conservation: a perspective for the management of phytogenetic resources}

\author{
Conservação in vitro: uma perspectiva para \\ a gestão dos recursos fitogenéticos
}

\begin{abstract}
Miguel Macgayver Bonilla Morales'1, Carolina Mancipe Murillo² \& Adriana Carolina Aguirre Morales ${ }^{3}$

'Licenciado en Producción Agropecuaria, Magister en Ciencias Biológicas con énfasis en Recursos Fitogenéticos Neotropicales. ${ }^{2}$ Ingeniera agrónoma. ${ }^{3}$ Ingeniera Agrónoma, Candidata a Magister en Ciencias Biológicas con énfasis en Recursos Fitogenéticos Neotropicales.

${ }^{1,3}$ Departamento de Ciencias Biológicas. Facultad de Ciencias Agropecuarias. Universidad Nacional de Colombia Sede Palmira. Palmira. Colombia. 2Jardín Botánico de Bogotá José Celestino Mutis. Bogotá. Colombia.

1mmbonillam@unal.edu.co, ${ }^{2}$ cmancipem@unal.edu.co, ${ }^{3}$ adcaguirremo@unal.edu.co
\end{abstract}

\section{Resumen}

Los recursos fitogenéticos comprenden la diversidad vegetal cultivada que tiene un valor real o potencial en la seguridad alimentaria de la humanidad. Desde esta perspectiva, cada una de las especies que han presentado un proceso de domesticación tanto a nivel local o mundial, asociando sus parientes silvestres, poseen un patrimonio genético invaluable. Por tal motivo, las especies cultivadas pueden beneficiarse mediante el flujo genético de sus parientes en busca de resistencia a plagas y enfermedades, fitomejoramiento o material adaptado al cambio climático. De tal manera, la conservación del germoplasma que posee una especie es prioridad para mantener la variabilidad genética inter e intra-especifica, así como la de sus accesiones. Las estrategias de conservación ex situ, en campo, presentan el riesgo de pérdida por condiciones climáticas adversas, ataque de agentes patógenos, altos costos asociados al manejo agronómico, preparación de terreno, insumos y dificultad del manejo e intercambio de material.
No obstante, las técnicas de cultivo de tejidos vegetales permiten mantener las plántulas en bancos de germoplasma in vitro, libres de patógenos, en espacio reducido, a bajo costo y condiciones controladas que facilitan el manejo a corto y largo plazo de material vegetal, particularmente, de especies con propagación vegetativa. Esta investigación tuvo como objetivo determinar los componentes claves para introducir especies cultivadas y establecer bancos de germoplasma in vitro que faciliten el intercambio de material vegetal entre entidades y personas, para garantizar la conservación, disponibilidad y uso de la diversidad genética obtenida por los cultivadores.

Palabras claves: agrobiodiversidad, conservación ex situ, cultivo in vitro, diversidad cultivada.

\section{Abstract}

Phytogenetic resources include the cultivated vegetable diversity which has a real or potential value 
in the food security of humanity. From this perspective, each of the species presented a domestication process either local or global, involving their wild relatives, they possess an invaluable genetic heritage. For this reason, cultivated species can benefit through gene flow from relatives in search of resistance to pests and diseases, breeding or material adapted to climate change. So, the conservation of germplasm is a priority to keep the variability genetics inter and intra - specified, as well as of their accessions. Strategies of conservation ex-situ in field, run the risk of loss due to adverse climatic conditions, attack of pathogenic agents, high costs associated with agronomic management, preparation of land, inputs and difficulty of the management and exchange of material. However, plant tissue culture techniques allow to keep the seedlings in gene banks in-vitro, free of pathogens, footprint, low cost and controlled conditions that facilitate the handling short and long term of plant material, particularly species with vegetative propagation. This research aimed to determine the key components to enter cultivated species and establish in-vitro germplasm banks that facilitate the exchange of plant material between entities and individuals, to ensure the preservation, availability and use of genetic diversity obtained by growers.

Keywords: agro-biodiversity, conservation ex-situ, in-vitro culture, cultivated diversity

\section{Resumo}

Os recursos fitogenéticos compreendem a diversidade vegetal cultivada que tem um valor real o potencial na segurança alimentar da humanidade.
Desde esta perspectiva, cada uma das espécies que têm apresentado um processo de domesticação tanto a nível local o mundial, associando os seus parentes selvagens, possuem um patrimônio genético inestimável. Por tal motivo, as espécies cultivadas podem se beneficiar através do fluxo gênico dos seus parentes em busca de resistência a pragas e doenças, melhoramento vegetal ou material adaptado às mudanças climáticas. Desse modo, a conservação do germoplasma que possui uma espécie é de vital importância para manter a variabilidade genética inter e intra-específica, bem como a dos seus acessos. As estratégias de conservação ex situ, em campo, têm como risco a perda por condições climáticas adversas, o ataque de agentes patógenos, altos custos associados ao manejo agronômico, preparação da área, insumos e dificuldade de manejo e intercambio de material. No obstante, as técnicas de cultura de tecidos vegetais permitem manter as plântulas em bancos de germoplasma in vitro, livres de patógenos, em espaço reduzido, baixo custo e condições controladas que facilitam o manejo a curto e largo prazo do material vegetal, especialmente, de espécies com propagação vegetativa. Esta pesquisa teve como objetivo determinar os componentes chaves para introduzir espécies cultivadas e estabelecer bancos de germoplasma in vitro que facilitem 0 intercambio de material vegetal entre entidades e pessoas, para garantir a conservação, disponibilidade e uso da biodiversidade genética obtida pelos produtores.

Palavras-chave: agrobiodiversidade, conservação ex situ, cultura in vitro, diversidade cultivada

\section{Introducción}

La población humana está aumentando y consecuentemente, la demanda de alimentos, especialmente en países desarrollados (Radovet \& Cahita, 2012). De una cifra actual que supera los 7 mil millones habitantes se espera para el 2050 un incremento de cerca del 30\% alcanzando en sólo
25 años alrededor de 9 a 10 mil millones (Calva \& Pérez, 2005). Los recursos fitogenéticos son considerados la base de la seguridad alimentaria, de ahí la importancia de mantener la diversidad genética de las plantas silvestres, tradicionales, regionales y cultivos mejorados (FAO, 2010; Hidalgo \& 
Vallejo, 2014). La conservación de estos recursos abarca métodos que capten la mayor cantidad de diversidad de genotipos, así como uso de técnicas de conservación y posterior multiplicación que mitiguen su pérdida a través del tiempo, de forma controlada, buscando maximizar la diversidad de ejemplares recolectados de poblaciones en campo o en su centros de origen (Sánchez \& Jiménez, 2010; Rivera et al., 2008).

La técnica de cultivo de tejidos vegetales a través de la propagación in vitro permite la producción masiva de plantas libre de patógenos, a bajo costo, en espacio reducido, en menor tiempo, bajo condiciones controladas con enfoques comerciales y agroindustriales, además de la producción de material vegetal para el establecimiento de cultivos y la generación de metabolitos secundarios (Bonilla \& Hernández, 2012; Tavazza et al., 2015). También, abre alternativas para el establecimiento de bancos de germoplasma para la conservación in vitro e intercambio de recursos fitogenéticos (FAO, 2010; Bonilla et al., 2012). Los bancos de germoplasma in vitro incluyen accesiones de especies que presentan semillas de corta y poca viabilidad, enfocados particularmente a cultivos de propagación vegetativa, que son altamente heterocigóticos y requieren multiplicación clonal para conservar su integridad genética. Este último componente incorpora raíces y tubérculos de corta vida en el proceso de almacenamiento, ejemplo, Solanum tuberosum L. (papa), Ipomoea batata L. (boniato) y Manihot esculenta Crantz (yuca) como otras especies que hacen parte de la diversidad vegetal cultivada utilizada principalmente en la alimentación humana (García, Feria \& Acosta, 2007; Bonilla \& Caetano, 2013).

La agrobiodiversidad se expone diariamente a factores como el cambio climático, aumento en la demanda de los recursos naturales, minería, explotación de hidrocarburos, ampliación de la frontera agrícola, contaminación industrial, deforestación, pérdida de la diversidad genética, erosión del suelo como de agroecosistemas (Sans, 2007; González y Martín, 2011; Mohan, 2011;
Reed et al., 2011), importantes para su desarrollo, crecimiento y permanencia presente y futura. Por tal motivo, la estrategia mundial para la conservación de plantas, firmada por más de 180 países en 2002, plantearon frenar esta pérdida mediante la comprensión y conservación de la diversidad vegetal, y la promoción del uso sostenible mediante técnicas de conservación en campo y laboratorio (Mohan, 2011; Reed et al., 2011). Por lo tanto, el presente artículo tiene como objetivo determinar los principales procesos para introducir especies cultivadas en bancos de germoplasma in vitro que faciliten el intercambio de material vegetal entre entidades y personas, en busca de garantizar la conservación, disponibilidad y uso de la diversidad genética obtenida por los cultivadores.

\section{Conservación in vitro}

En general, las técnicas de conservación se han centrado en plantas vasculares con semillas (Simpson, 2010). El cultivo in vitro se remonta al desarrollo de técnicas de conservación, siendo estas relativamente fáciles (Martín, 2006; Rowntree et al., 2011). De acuerdo con Mohan (2011) e Iriondo (2011), la conservación in vitro trae una serie de ventajas, como la obtención de material libre de patógenos, tasas de multiplicación altas, suministro constante de plantas a los productores, bajo costo en la producción, mantenimiento de la fidelidad genética del material. Sin embargo, algunas desventajas pueden ser la pérdida de material genético por contaminaciones, dependencia de mano de obra calificada y que el material puede destruirse en caso de un siniestro.

El conocimiento de la composición genética es esencial en cualquier plan de conservación integral, siendo esta un componente importante en la biología de la conservación por ser el nivel más alto de diversidad biológica, pues sin variación genética las poblaciones no podrían evolucionar y adaptarse a los cambios ambientales (Gonzales \& Martin, 2011; Rowntree et al., 2011). Es por esto que cuando una especie es conservada in vitro debe revisarse de manera periódica y evitar los 
ciclos muy largos para desfavorecer las variaciones somaclonales (Gonzales \& Martin, 2011).

La conservación de tejidos prolongada puede resultar en cambios genéticos y dar lugar a una progenie con alteraciones características (variación somaclonal) en lugar de conservar las adaptaciones genotípicas a sus condiciones naturales. Sin embargo, a diferencia de otros métodos de conservación (bancos de semillas y colecciones vivas en campo) los bancos de genes in vitro contienen todo la variabilidad genética de plantas que pueden ser continuamente propagadas y estar disponibles para investigación, así mismo, provee clones en caso de necesitar material homogéneo en investigaciones y posibilidad de cultivar materiales élite, libre de patógenos (Daskalova et al., 2011; García, Feria \& Acosta, 2007).

Para la conservación de tejidos en frío, generalmente se recurre a las zonas meristemáticas, que se encuentran en activa división y que son más resistentes a las bajas temperaturas dado su tamaño pequeño, citoplasma denso y pocas vacuolas, razón por la cuales el contenido de agua intracelular es bajo, aspecto importante a tener en cuenta para evitar daños durante el proceso de congelación (García, Feria \& Acosta, 2007), adicionalmente deben tenerse en cuenta las condiciones de temperatura a las cuales la planta es tolerable. Las especies tropicales son sensibles al frío y deben ser almacenadas a temperaturas relativamente altas en función de su sensibilidad, por ejemplo, el género Musa sp., se almacena preferiblemente a $15^{\circ} \mathrm{C}$ durante máximo 15 meses tiempo en el cual debe ser transferido a campo. Por su parte, la yuca puede ser conservada a temperaturas mayores o iguales a $20^{\circ} \mathrm{C}$ (Engelmann, 2011). En estos casos la reducción de la temperatura puede realizarse al disminuir la intensidad de luz o al realizar cultivo en oscuridad.

Los métodos de conservación in vitro se consideran particularmente interesantes para aquellas especies hortícolas con semillas recalcitrantes o propagadas por métodos vegetativos, por eso, la yuca es una de las especies que más se ha potenciado mediante el uso de esta técnica (Figura 1). Igualmente ocurre con la mayoría de las rosas, las cuales al ser heterocigotas y muy variables, se propagan por métodos vegetativos. En Irán un gran número de especies de rosa han sido expuestas a la extinción y por lo tanto las técnica de conservación son necesarias para preservarlas (Kavand et al., 2011).

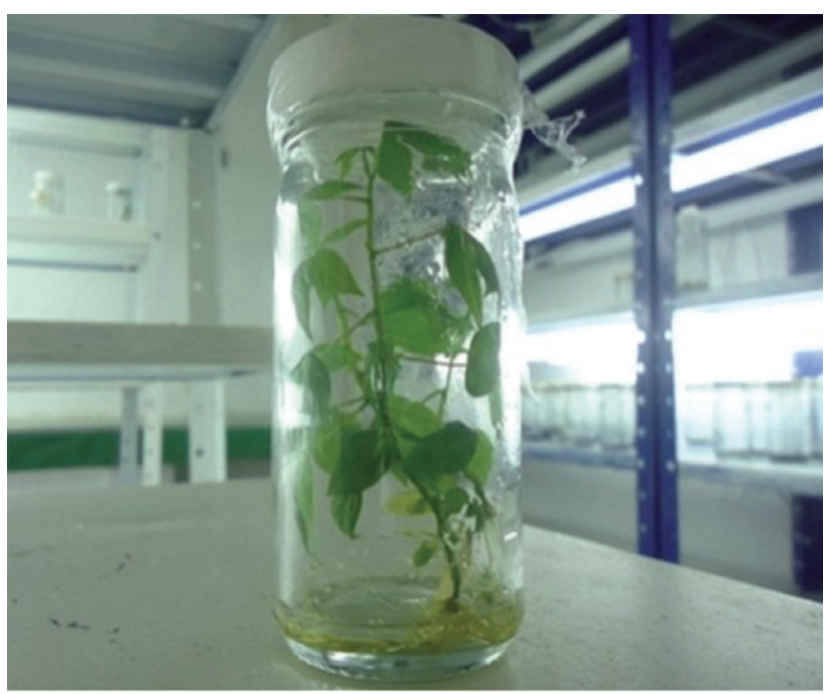

Figura 1. Conservación in vitro de yuca (Manihot esculenta).

En general la conservación puede ser clasificada de acuerdo con su duración: a corto plazo (crecimiento reducido,) y a largo plazo (crioconservación) (Sánchez \& Jiménez, 2010). De acuerdo con Engelmann (2011) y Pence (2011), existen cuatro razones por las cuales se deben emplear las técnicas de propagación y conservación in vitro: i) algunas plantas no producen semillas y como es el caso del plátano y el banano, se reproducen de forma vegetativa, ii) algunas especies generan genotipos estériles y genotipos con semilla ortodoxa, altamente heterocigotas por lo cual es preferible conservarlas como clones, como la papa y caña de azúcar. iii) especies frutales y forestales tropicales generalmente producen semillas recalcitrantes, iv) la conservación de especies con semilla intermedias es aún problemática. 
Especies en peligro de extinción y que prestan servicios al hombre tales como alimentación, insumos para construcción, salud y en muchos casos ornamentales merecen particular atención y esfuerzos de conservación (Bonilla \& Caetano, 2014). Zonas ricas en biodiversidad proveen todos estos beneficios, además de los servicios ambientales, sin embargo el cambio en el uso de estos ambientes principalmente para la agricultura (caña de azúcar, caucho y palma de aceite) contribuyen a la pérdida de biodiversidad y de recursos genéticos potenciales tal como es el caso de la cuenca del Amazonas, Papua, Nueva Guinea y los bosques de Indonesia (Reed et al., 2011).

Entre los recursos fitogenéticos ornamentales, las orquídeas, una de las familias más grandes y diversas de plantas con flores, son colectadas de manera desaforada, pierden hábitats y sufren los impactos del cambio climático (Aguirre, Mancipe \& Bonilla, 2015). Los complejos requisitos de polinización, germinación de las semillas, y otras factores bióticos y abióticos contribuyen con la pérdida de plantas cuando no se cumple con estas condiciones, por lo cual toma importancia la conservación in vitro de plantas ornamentales (Hidalgo \& Vallejo, 2014). Por lo anterior, la conservación de orquídeas debe integrar las amenazas actuales y futuras, polinización, biología reproductiva, propagación in vitro y ex situ, mantenimiento de diversidad genética como estrategia de recuperación de especies (Reed et al., 2011). Las orquídeas son bien conocidas como importantes plantas ornamentales en Tailandia, especialmente las orquídeas silvestres, en el caso de Cleisostoma arietinum es una orquídea epifita y nativa de Tailandia, una especie en peligro de extinción que podría ser conservada, mediante la evaluación de protocolos de conservación (Maneerattanarungroj, Bunnag \& Monthatong, 2007).

Lima et al. (2011) determinaron mediante experimentación que la técnica de conservación in vitro en siempreviva (Syngonanthus mucugen- sis) es viable utilizando un medio de cultivo $1 / 2$ MS, $15 \mathrm{gL}^{-1}$ de sacarosa a $18^{\circ} \mathrm{C}$, pues posibilita la conservación de la especie hasta por 180 días sin subcultivo, lo cual es importante dado que la especie es una herbácea ornamental que se encuentra en peligro por la explotación extractivista de sus flores. Además, procesos de limpieza de agentes perjudiciales para las plantas pueden se erradicados mediante el cultivo in vitro, que juega un papel importante en la conservación de material élite, con características de resistencia al patógeno, facilitando posteriores multiplicaciones (Shukla et al., 2012)

Generalmente, se busca establecer protocolos de propagación y conservación de acuerdo a la especie, tal como ocurre con Morinda reticulata, planta medicinal de la India amenaza por su uso indiscriminado (Raveendran et al., 2012). Con fines de conservación Raveendran et al., (2012) diseñaron un protocolo para la propagación plantas a partir segmentos nodales, siendo este importante para su conservación y restauración. Por su parte, Haberlea rhodopensis es una planta balcánica capaz de sobrevivir bajo deshidratación casi completa (anabiosis), con una actividad metabólica muy baja y tras ser hidratada vuelve rápidamente a la condición fisiología normal, dado su interés y uso potencial de sus capacidades genéticas como alternativa al cambio climático, Daskalova et al. (2011) realizaron estudios de propagación de la especie utilizando la combinación de tecnologías de conservación en campo e in vitro, finalmente se llevó a cabo el establecimiento de un banco in vitro.

La micropropagación, cultivo de tejidos y conservación in vitro y ex situ de platas medicinales satisface la creciente demanda por la medicina naturista y a través del cultivo reduce la presión sobre las poblaciones naturales, siendo una de las maneras más eficaces de conservación (Krishnan, Decruse \& Radha, 2011). Las plantas medicinales albergan gran diversidad de propiedades para el tratamiento de múltiples 
dolencias, esta diversidad representa un importante potencial en la industria de productos medicinales naturales o en la industria farmacéutica (Bonilla \& Caetano, 2014). Aunque el descubrimiento de fármacos a partir de plantas medicinales continúa, se presentan restricciones con la disponibilidad de las plantas, para ello se han utilizado técnicas de conservación (Krishnan, Decruse \& Radha, 2011). Se han desarrollado protocolos de regeneración in vitro para especie de importancia farmacológica como Passiflora foetida L., con la finalidad de multiplicar el material vegetal existente y obtener líneas o clones ideales para el abastecimiento de los compuestos deseados (Shekhawat et al., 2015).

Otras especies son importantes como fuente de genes, como el caso de las especies silvestres de Arachis, importante fuente de genes para la mejora de cacahuate. Su conservación en bancos requieren de renovaciones continuas sin embargo mucho de estos materiales se pierden por contaminación o disminución en la viabilidad que conducen a pérdidas de accesiones enteras (Gagliardi et al., 2007). Igualmente, especies de Vanilla (Orquidaceae) son importantes por su fuente de Vanillinin, utilizado como saborizante, para potenciar el aprovechamiento del género se han desarrollado programas para su conservación in vitro con la intención de preservar y permitir el intercambio de los recursos genéticos (Divakaran, Nirmal \& Peter, 2006).

De este modo, las técnicas de laboratorio permiten mantener la integridad genética, promover altas tasas de multiplicación en espacio reducido en condiciones asépticas y permitir el intercambio de germoplasma (Lima et al., 2011). Consiste en técnicas de crecimiento lento y conservación a corto, mediano y largo plazo (Normah et al., 2011; Pence,
2011). Así mismo las técnicas in vitro se complementan con las in situ y tienen un respaldo con la conservación en bancos genéticos (Guo, Stiles \& Liu, 2013; García, Feria \& Acosta, 2007; Reed et al., 2011; Jung et al., 2012; Kaviani, Darabi \& Roudposhti, 2012; Pence, 2011).

\section{Conservación a corto plazo}

En este tipo de conservación los explantes permanecen por máximo 6 meses en cultivo in vitro, tiempo en el cual se retrasa el crecimiento y se disminuyen los intervalos de subcultivo, mediante la reducción en la temperatura en los cuartos de crecimiento y las modificaciones en el medio de cultivo, tal como la disminución del contenido mineral (Rayas et al., 2002), la adición de agentes osmóticos activos y/o la incorporación de retardadores de crecimiento (García, Feria \& Acosta, 2007). Esta reducción disminuye la actividad metabólica y por ende el crecimiento de los explantes, y es combinada con otros factores de acuerdo a la especie para lograr la reducción del crecimiento; entre ellos la intensidad lumínica y disminución en la disponibilidad de oxigeno (Sánchez \& Jiménez, 2010; Normah et al., 201; Lima et al., 2011). Tiene como objetivos incrementar la longevidad in vitro de los cultivos sin que se produzcan cambios genéticos, por tanto, no hay una detención total de los procesos celulares sino una disminución en la velocidad en que ocurren los mismos y para reducir la frecuencia de transferencia de las plantas a medio de cultivo fresco (Figura 2). La limitación del crecimiento por efecto de la concentración osmótica se debe a la reducción de la adsorción de agua y nutrientes del medio de cultivo (García, Feria \& Acosta, 2007). La sacarosa como es altamente metabolizable, puede actuar como agente osmótico en concentraciones elevadas (Ramírez, Guevara \& Escobar, 2012). 


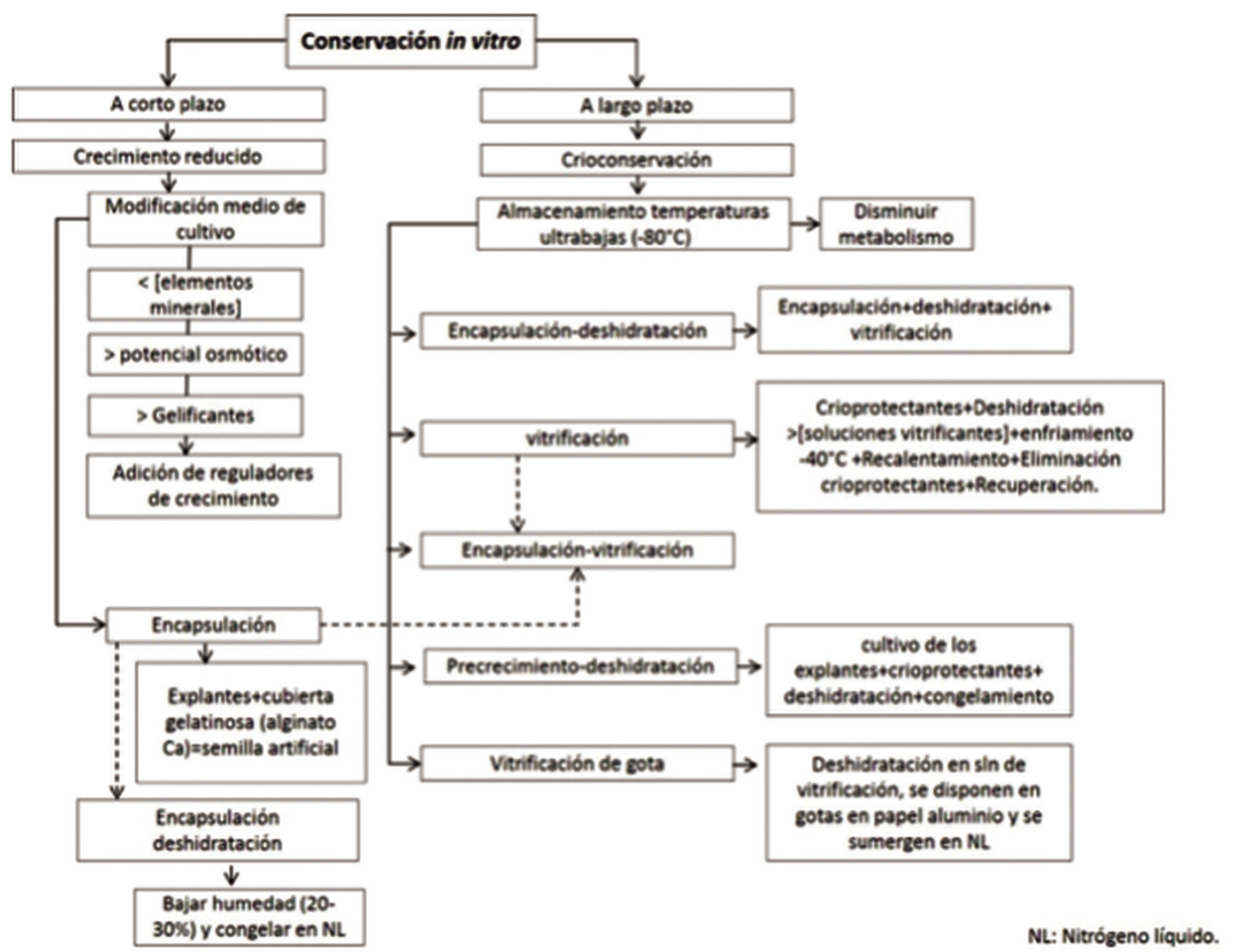

Figura 2. Estrategia de conservación in vitro para los recursos fitogenéticos.

Otros agentes osmóticos no metabolizables, como el manitol y el sorbitol, son más efectivos en la limitación del crecimiento, porque interactúan con el contenido de sacarosa y la temperatura de conservación (García, Feria \& Acosta, 2007). Eventualmente en los cultivos conservados in vitro, debido a las bajas temperaturas, la alta concentración osmótica, la reducción de la concentración de sales inorgánicas y la presencia de inhibidores del crecimiento, se puede producir un oscurecimiento de los tejidos, seguido de defoliación. Esto se atribuye a la oxidación fenólica y a la inducción de senescencia por la presencia de determinadas concentraciones de gases como el etileno, especialmente cuando se utilizan frascos pequeños o han sido sellados herméticamente (Sánchez \& Jiménez, 2010).
Dependiendo de la principal fuente de alimento usada por los países, la investigación como los programas de conservación in vitro se centran en el estudio de sus recursos, ejemplo Cuba ha avanzado particularmente en ñame (Dioscorea spp), cacao (Theobroma cacao L.) y boniato o batata (Ipomea batata), y algunas plantas medicinales (Silva, 2001). Aunque, existen otras especies que tienen prioridad dentro de los recursos fitogenéticos para su conservación in vitro de los géneros Agave, Allium, Ananas, Canna, Colocasia, Ficus, Ipomea, Musa, Olea, Piper, Saccharum, Solanum, Vanilla, vitis, Xanthosoma, tubérculos andinos como frutales, entre otros (Roca, Arias \& Chávez, 1991; Scott, Rosegrant \& Ringler, 2000; Matos et al., 2015). En la Tabla 1 se encuentra una lista de los recursos genéticos vegetales que son principalmente cultivados y conservados en bancos de germoplasma. 
Tabla 1. Conservación in vitro de algunas especies vegetales cultivadas en bancos de germoplasma.

\begin{tabular}{|c|c|c|c|c|}
\hline Familia & Especie & $\begin{array}{l}\text { Nombre } \\
\text { vulgar }\end{array}$ & Institución & País \\
\hline \multirow[t]{3}{*}{ Amaryllidaceae } & Allium cepa $L$. & $\begin{array}{l}\text { Cebolla } \\
\text { cabezona }\end{array}$ & NBPGR & India \\
\hline & Allium sativum $L$. & Ajo & NBPGR & India \\
\hline & Allium fistulosum $L$. & $\begin{array}{l}\text { Cebolla } \\
\text { larga }\end{array}$ & NBPGR & India \\
\hline \multirow[t]{2}{*}{ Rutaceae } & Citrus spp & Citrus & CITRICULTURA & Brasil \\
\hline & & & PROINPA & Bolivia \\
\hline Araceae & Xanthosoma spp & Bore & INIVIT & Cuba \\
\hline Araceae & Colocasia esculenta & Malanga & INIVIT & Cuba \\
\hline Asteraceae & $\begin{array}{l}\text { Smallanthus sonchifolius } \\
\text { (Poepp. \& Endl.) H. Robinson }\end{array}$ & Yacon & CIP & Perú \\
\hline \multirow[t]{2}{*}{ Basellaceae } & Ullucus tuberosus Caldas & Ulluco & CIP & Perú \\
\hline & & & PROINPA & Bolivia \\
\hline Brassicaceae & Lepidium meyenii Walp. & Maca & CIP & Perú \\
\hline Bromeliaceae & Ananas comosus & Piña & ISACA & Cuba \\
\hline \multirow[t]{2}{*}{ Cannaceae } & Canna edulis Ker Gawl. & Achira & CIP & Perú \\
\hline & & & PROINPA & Bolivia \\
\hline \multirow[t]{2}{*}{ Convolvulaceae } & Ipomea batatas (L.) Lam. & Papa dulce & CIP & Perú \\
\hline & & & INIVIT & Cuba \\
\hline Dioscoraceae & Dioscorea spp & Ñame & IITA & Nigeria \\
\hline \multirow{2}{*}{ Euphorbiaceae } & Manihot esculenta Crantz & Yurg & CIAT & Colombia \\
\hline & Manihot spp. & yuca & INIA & Venezuela \\
\hline \multirow[t]{2}{*}{ Fabaceae } & Pachyrhizus ahipa (Wedd.) & Maca & CIP & Perú \\
\hline & & & PROINPA & Bolivia \\
\hline Lauraceae & Persea americana Mill. & Aguacate & INIFAP & México \\
\hline Musaceae & Musa spp & Banana & ITC-INIBAP & Bélgica \\
\hline Malvaceae & Theobroma cacao $L$. & Cacao & INTA & Nicaragua \\
\hline Nyctaginaceae & Mirabilis expansa & Mauka & CIP & Perú \\
\hline \multirow[t]{2}{*}{ Oxalidaceae } & Oxalis tuberosa Molina & Oca & CIP & Perú \\
\hline & & & PROINPA & Bolivia \\
\hline \multirow[t]{2}{*}{ Passifloraceae } & Passiflora spp. & $\begin{array}{l}\text { Frutas de la } \\
\text { pasión }\end{array}$ & EMBRPA & Brasil \\
\hline & & & JBB & Colombia \\
\hline \multirow[t]{2}{*}{ Poaceae } & Saccharum officinarum $L$. & $\begin{array}{l}\text { Caña de } \\
\text { azúcar }\end{array}$ & WICSCBS & Barbados \\
\hline & & & INICA & Cuba \\
\hline Rubiaceae & Coffea spp & Café & PROCAFE & Salvador \\
\hline Rutaceae & Citrus spp & Citrus & CITRICULTURA & Brasil \\
\hline \multirow[t]{4}{*}{ Solanaceae } & Solanum tuberosum L. & Papa & CIP & Perú \\
\hline & & & PROINPA & Bolivia \\
\hline & Solanum phureja Juz. \& Bukasov & Papa criolla & CIP & Perú \\
\hline & & & PROINPA & Bolivia \\
\hline \multirow[t]{2}{*}{ Tropaeolaceae } & Tropaeolum tuberosum Ruiz \& Pavón & Mashua & CIP & Perú \\
\hline & & IVIasmua & PROINPA & Bolivia \\
\hline
\end{tabular}




\section{Condiciones de incubación}

\section{a. Medio de cultivo}

La reducción en la concentración de elementos minerales (carbohidratos metabolizables) en el medio de cultivo, es una estrategia importante para la reducción del crecimiento del explante, así mismo se puede aumentar el potencial osmótico del medio mediante el uso de carbohidratos no metabolizables, tales como el manitol, mayor concentración de gelificantes y adición/supresión de reguladores de crecimiento, por lo anterior el explante absorbe los nutrientes de manera lenta reduciéndose consecuentemente el crecimiento (Sánchez \& Jiménez, 2010; Lima et al., 2011; Engelmann, 2011).

En estudios realizados por Kavand et al., (2011), se demostró que la inclusión de $3 \mu \mathrm{M} \mathrm{GA}_{3}$ en el medio de cultivo, incremento significativamente el número de brotes axilares y la altura de los tallos. De acuerdo con autores para rosa se recomienda una concentración de auxinas ( $1 \mathrm{mg} / \mathrm{L}$ de IBA y $0,5 \mathrm{mg} / \mathrm{L}$ de IAA). Numerosos parámetros influyen en la eficiencia de ensayos in vitro: protocolos de almacenamiento de crecimiento lento como el tipo de explantes, su estado fisiológico al entrar en el almacenamiento, el tipo de recipiente de cultivo, su volumen, así como el tipo de cierre del recipiente de cultivo (Engelmann, 2011).

\section{b. Encapsulación}

Las plantas propagadas de manera in vitro pueden estar expuestas a deterioro en comercialización, inconvenientes superados con el uso de la técnica de encapsulación (Tavazza et al., 2015). Este material ha sido descrito como un embrión somático encapsulado, o semilla sintética contenido en una matriz, entendiéndose como semilla a cualquier tipo de propágulo vegetativo. El producto del proceso de encapsulación es un propágulo (unipolar o bipolar) en una matriz para proteger de daños mecánicos y fisiológicos, convirtiéndose en un mecanismo importante en el intercambio de material libre de patógenos entre laboratorios (Germana et al., 2007).
Esta técnica se basa en recubrir los embriones somáticos, yemas gametofíticas o ápices con una cubierta gelatinosa (por ejemplo alginato de calcio) para formar semillas sintéticas, proporcionando a la vez protección contra la deshidratación y las bajas temperaturas durante el almacenamiento (Sánchez \& Jiménez, 2010; Barnicoat et al., 2011; Engelmann, 2011; Bunnag \& Khonkayan, 2010). En el método de encapsulación los explantes son recubiertos por perlas de alginato de calcio (que puede contener sales minerales y sustancias orgánicas), formando de esa manera las semillas artificiales. Posteriormente estas semillas son tratadas con altas concentraciones de sacarosa y se secan dejando un contenido de humedad de 20 a $30 \%$ (bajo flujo de aire o el uso de gel de sílice) para luego ser congelado rápidamente en nitrógeno líquido. Debido a las altas tensiones provocadas durante el proceso, se debe utilizar crioprotectantes como la sacarosa, que aumenta la tolerancia a desecación ayudando a mantener la viabilidad de los tejidos (Kaviani, Darabi \& Roudposhti, 2012; Faisal et al., 2006).

La deshidratación permite mayor flexibilidad cuando se manipula gran número de muestras y no se requiere de congeladores programables, de esta manera, el método de encapsulación y deshidratación ha sido aplicado a los ápices (tanto de clima templado y especies tropicales), semillas, embriones somáticos y suspensiones celulares en un gran número de especies (Roca, Arias \& Chávez, 1991; Ramírez, Guevara \& Escobar, 2012). En este método, la extracción gradual de agua a partir de explantes encapsulados es realizada durante el precultivo en medio enriquecido con sacarosa (Pimda \& Bunnag, 2010). Las molaridades de sacarosa en los granos se incrementa aún más por la tasa adicional de aire de secado o deshidratación con aire laminar en cabina, para alcanzar el punto de saturación de la solución de sacarosa y transición durante el enfriamiento a $-196^{\circ} \mathrm{C}$ en nitrógeno líquido, para prevenir la formación de cristales de hielo (Pimda \& Bunnag, 2010; Bunnag \& Khonkayan, 2010). 
Se han propuesto numerosos factores que participan en la adaptación de los explantes a la desecación y bajo estrés térmico. La sacarosa, glucosa, fructosa y oligosacáridos (por ejemplo, rafinosa y estaquiosa) se han reportado como un factor principal que se encuentra comúnmente en la acumulación de azúcares solubles junto con el desarrollo de la tolerancia a la congelación en la mayoría de las plantas, lo que sugiere que estos azúcares no sólo sirven como osmoprotectores, sino que también juegan un papel en la protección de la membrana celular de los daños causados por la deshidratación y la congelación a través de la interacción con la bicapa lipídica (Pimda \& Bunnag, 2010).

Además de los azúcares, el ácido abscísico (ABA) también ha sido propuesto para desempeñar un papel en la baja temperatura y tolerancia a la sequía (Azcón-Bieto \& Talon, 2008). En el endurecimiento de las plantas se exhibe un aumento endógeno de ABA, lo que implica que esta fitohormona está posiblemente asociada con el consiguiente aumento de la tolerancia a la congelación de las plantas expuestas a bajas temperaturas, razón por la cual el $\mathrm{ABA}$ ha sido empleado en mejoramiento, en la desecación y en la tolerancia a temperaturas bajas en muchas especies (Pimda \& Bunnag, 2010; Bunnag \& Khonkayan, 2010).

En algunos casos, la encapsulación de segmentos nodales de especies en peligro de extinción como Tecomella undulata mediante una matriz de gelificante ( $3 \%$ de Alginato de Sodio en MS basal) y suplementado con 2,5 M BA (6- Bencyl Aminopurina) + 0,5 M AIA (3 Acido Indoloacético), seguido de su plantación en MS +10 M BA permite una alta producción de brotes después del proceso de almacenamiento (Shaheen \& Shahzad, 2015). Sin embargo, especies arbóreas de larga vida como las Sequoia sempervirens bajo procesos de no encapsulación de yemas durante 12 meses, responde idóneamente a la regeneración de brotes (Ozudogru et al., 2011).

\section{Conservación a largo plazo}

Esta técnica permite almacenar semillas, meristemos, ápices, polen, callos y suspensiones celulares, considerándose una herramienta útil para evitar variaciones somaclonales en plantas con propagación vegetativa y permitiendo la conservación de explantes por tiempo de almacenamiento indefinido (Sánchez \& Jiménez, 2010). También se le ha denominado a este proceso crecimiento lento, por lo que mediante regulación osmótica se controla la entrada de compuestos químicos indispensables para el crecimiento y desarrollo para la planta (Tavazza et al., 2015).

\section{Crioconservación}

La crioconservación se puede considerar un sistema no letal de almacenamiento de tejidos biológicos a temperaturas ultra bajas, pues permite detener su metabolismo (Rivera et al., 2008; Tavazza et al., 2015). Por consiguiente, las estructuras vegetales utilizadas generalmente son meristemos, ápices, semillas, embriones cigóticos, polen y embriones somáticos para asegurar la estabilidad genética de los materiales (Rivera et al., 2008). El almacenamiento durante periodos prolongados de células, tejidos u órganos vegetales vivos a temperaturas extremadamente bajas $\left(-80^{\circ} \mathrm{C}\right)$, utilizando nitrógeno líquido, el cual normalmente se encuentra a $-196{ }^{\circ} \mathrm{C}$ es una de las técnicas que principalmente se utilizan, aunque, en algunas ocasiones, se combina el nitrógeno líquido con otros gases inertes (como el helio y el argón), este detiene las actividades metabólicas del explante y a la vez permite su viabilidad sin que existan alteraciones fisiológicas durante el almacenamiento y manteniendo las características genéticas (Sánchez \& Jiménez, 2010; Krishnan, Decruse \& Radha 2011; Guo, Stiles \& Liu, 2013; García, Feria \& Acosta, 2007).

Entre las especies crioconservadas se encuentran plátano (Musa spp.), yuca (Manihot esculenta), zarza fruta (Rubus), pera (Pyrus), solanáceas, café (Coffea arabica), palma de aceite (Elaeis 
Guineensis), té (Camellia sinensis) (Mohan, 2011), Rubus, Pyrus, Solanum, Elaeis guineensis (Rivera et al., 2008) y es una alternativa para la conservación de especies como Vanda pumila, Bletilla striata, Geodorum densiflorun, Doritaenopsis orchid, Ipomoea batatas y Armoracia rusticata (Maneerattanarungroj, Bunnag \& Monthatong, 2007). La crioconservación de semillas reclacitrantes es exitosa principalmente en cítricos entre ellos $C$. aurantifolia y $C$. limonia que de acuerdo con ensayos el $91.7 \%$ de las semillas sobrevivió a la técnica (Normah et al., 2011; Reed et al., 2011).

En la vitrificación se ofrecen ventajas como la rápida congelación, lo que es más apropiado para órganos, en estos casos, la etapa crítica para la supervivencia es la deshidratación y no la congelación, por lo que se sumerge el material en nitrógeno para impedir la formación de cristales de hielo a fin de evitar daños que imposibiliten la regeneración en el tejido al descongelar el material crioconservado (Engelmann, 2011; Pence, 2011). Así mismo tiene aplicaciones en la eliminación de virus (crioterapia), al actuar sólo sobre el domo meristematico y en la base del primordio, lo que la convierte en una alternativa para su eliminación, como es el caso del virus del mosaico del pepino, virus del rayado del banano, virus del enrollamiento de la hoja de papa, entre otros (Mohan, 2011). Hasta la fecha, los patógenos graves en banano (Musa spp.), Citrus spp, vid (Vitis vinifera), Prunus spp., frambuesa (Rubus idaeus), papa (Solanum tuberosum) y batata (Ipomoea batatas) han sido erradicado mediante crioterapia (Engelmann, 2011).

Guo, Stiles \& Liu (2013) realizaron un estudio en Saussurea involucrata una planta medicinal de China, difícil de cultivar, pero mediante un método de optimización que consistió en la incubación por un periodo de 3 días en un medio que contenía $0.3 \mathrm{M}$ de sacarosa, se transfirió la planta a una solución de vitrificación durante 60 minutos y de inmediato se introdujeron en nitrógeno líquido. Para descongelar los tejidos se calentaron a $40^{\circ} \mathrm{C}$ durante 2 minutos, este método dio lugar a una supervivencia de $56 \%$ y una tasa de regeneración del $40 \%$, la regeneración se mejoró al incubar los callos a $4^{\circ} \mathrm{C}$ durante 12 días antes de iniciar el protocolo de crioconservación, aumentando la tasa de supervivencia del tejido a $75 \%$ y una tasa de regeneración mayor al $60 \%$, además se duplico el número de brotes regenerados por explante (Guo et al., 2013). El crecimiento lento de genotipos de alcachofa (Cynara cardunculus var. scolymus L.) presentaron estabilidad genética al ser evaluados durante los 6 y 12 meses de desarrollo, con un porcentaje de supervivencia que llego hasta el $85 \%$, y todas estas plantas rebrotaron, tanto que a los dos meses las vitroplantas recuperaron su crecimiento potencial.

En el caso del café, también, se han desarrollado protocolos de crioconservación con duplicados en nitrógeno líquido con materiales de varios países de América Latina y el Caribe (Biodiversity international, 2011; Jung et al., 2012; Scocchi et al., 2004). Las criocolecciones no requieren la regeneración periódica para mantener el vigor del material conservado, contrario a los bancos de germoplasma de campo, lo que significa un aumento en los costos, convirtiéndose en un riesgo si se conservan especies en peligro de extinción, pues podrían extinguirse (Biodiversity international, 2011).

En el éxito de plantas crioconservadas se deben tener en cuenta aspectos como: estado fisiológico de la planta, precultivo, tratamientos crioprotectantes, velocidades de enfriamiento, recalentamiento y recuperación al medio, con el fin de tener plantas vigorosas del criogéno. Los meristemos suelen ser escogidos para conservación debido a las pequeñas y compactas células con pocas vacuolas, lo que facilita el tratamiento, es así como se ha tenido éxito en ápices de yuca, banano, papa y Parkia speciosa Hassk. En el caso de semillas recalcitrantes se dificulta su crioconservación debido a los grandes contenidos y variaciones de humedad, estructura y la variación intra e interespecifica de las especies. Por lo tanto en meristemos apicales producidos a partir de brotes adventicios generados a partir de segmentos nodales in 
vitro son más uniformes y ofrecen mejores explantes para la crioconservación (Normah et al., 2011).

Durante el proceso de crioconservación se produce una serie de tensiones en la planta, causando daño oxidativo por la producción de especies reactivas de oxigeno (ROS), por lo cual se deben adicionar antioxidantes en diferentes pasos de los protocolos (Normah et al., 2011). Estudios de proteómica en banano elucida que las diferencias en tolerancia a deshidratación durante procesos de crioconservación pueden deberse a isoformas específicas de enzimas implicados en el metabolismo energético, así mismo con proteínas asociadas a la adaptación al estrés, por lo cual es fundamental los estudios en proteómica para observar los cambios en las expresiones proteicas asociadas con la técnica (Normah et al., 2011).

\section{Vitrificación}

La vitrificación implica el tratamiento de las muestras con crioprotectores, deshidratación con altas concentraciones de soluciones vitrificantes, rápido enfriamiento, recalentamiento, eliminación de crioprotectores y la recuperación (Engelmann, 2011). Las sustancias crioprotectoras más utilizadas son dimetilsulfóxido (DMSO), manitol, sorbitol, sacarosa y polietilenglicol (PEG), estos tienen acción osmótica, pero algunos de ellos (por ejemplo DMSO) pueden entrar en las células y proteger la integridad celular durante la congelación (Rivera et al., 2008). Para la crioconservación de tejidos diferenciados como órganos, ápices, embriones cigóticos y somáticos, se han desarrollado nuevas técnicas durante los últimos años que consisten en extraer la mayor parte del agua por deshidratación física u osmótica de los explantes seguida por una congelación muy rápida que produce vitrificación de los solutos contenidos dentro de las células, es decir, la formación de una estructura vítrea amorfa sin que se presente la formación de cristales de hielo que son perjudiciales para la estructura celular (Roca, Arias \& Chávez, 1991; Ramírez, Guevara \& Escobar, 2012). Para la mayoría de los materiales, es conveniente hacer un enfriamiento lento $\left(0,5\right.$ a $\left.2^{\circ} \mathrm{C} / \mathrm{min}\right)$ hasta alcanzar aproximadamente los $-40^{\circ} \mathrm{C}$, y posteriormente realizar la inmersión rápida de muestras en nitrógeno líquido (Rivera et al., 2008).

Por otro lado, la encapsulación-vitrificación es una combinación de procedimientos de encapsulación, deshidratación y vitrificación en la que las diferentes muestras se encuentran encapsuladas en perlas de alginato y sometidos a congelación por vitrificación (Martín, 2006; Raveendran et al., 2012; Tavazza et al., 2015). La deshidratación consiste en eliminación de humedad del explante y luego congelarlo de forma directa mediante inmersión en nitrógeno líquido. La desecación se logra al someter los tejidos a una corriente de aire comprimido o gel de sílice (Maneerattanarungroj, Bunnag \& Monthatong, 2007; Bunnag \& Khonkayan, 2010). Generalmente, la supervivencia óptima se logra cuando las muestras se congelan con contenidos de agua de 10 y $20 \%$ (en base al peso en fresco), y el precrecimiento y deshidratación consiste en el cultivo de los explantes en presencia de crioprotectantes, deshidratado bajo flujo de aire laminar y posterior congelamiento (Engelmann, 2011).

La supervivencia es generalmente alta, pues alcanza el $100 \%$ en algunos casos, por ejemplo, Allium, el ñame y la papa, por lo que la regeneración es rápida y directa y no se observa formación de callos sólo en los casos en que la técnica no está optimizada (Roca, Arias \& Chávez, 1991; Ramírez, Guevara \& Escobar, 2012). Estos resultados se deben a que la zona meristemática de los ápices, de la cual se origina el crecimiento organizado se compone por una población relativa de células homogéneas que se dividen activamente con pequeñas vacuolas y un alto radio núcleo citoplasmático (Ramírez, Guevara \& Escobar, 2012). Otra de las razones del éxito es el desarrollo de buenos protocolos, permitiendo la obtención de material homogéneo en términos de tamaño, forma y composición, estado fisiológico aumentando las posibilidades de respuestas positivas (García, Feria \& Acosta, 2007). 
Una técnica de crioconservación denominada encapsulación/deshidratación se ha aplicado con éxito en más de 30 especies incluyendo varios genotipos de pera, manzana, caña de azúcar y papa, donde se utilizan los ápices y se encapsulan en perlas de alginato, pretratados en medio líquido que contiene una alta concentración de sacarosa, y parcialmente desecado antes congelación en nitrógeno líquido (Scocchi et al., 2004). Por otro lado, este procedimiento presenta un comportamiento diferencial de las especies al ser sometidas a procesos como la inmersión en nitrógeno líquido, asociados a la congelación y descongelación que actúan como una escarificación, resultando en una mayor permeabilidad y cambio en la fisiología de las semillas después del almacenamiento criogénico que resulta en mayor germinación (Kokowicz et al., 2011; Bunnag y Khonkayan, 2010). Cuando las semillas son grandes, por lo que no se pueden congelar de manera directa, la crioconservación es una opción, sin embargo, los embriones a menudo presentan sensibilidad a la desecación (Engelmann, 2011).

\section{Conclusiones}

Los recursos fitogenéticos son la principal fuente de alimentación que garantiza la seguridad alimentaria de la humanidad, de tal manera, el preservar la identidad genética de cada una de las variedades o culti-variedades es importante para su uso local o mundial, por lo que la conservación ex situ mediante las técnicas de cultivo de tejidos en los procesos de micropropagación de explantes como los ápices de plantas de propagación vegetativa, garantiza el mantenimiento del patrimonio genético de cada una de las accesiones que se introducen al banco de germoplasma in vitro.

La conservación in vitro, a largo plazo, permite mantener vitroplantas, mínimo durante un año, con la finalidad de disminuir los costos en el manejo de material como el ciclo de subcultivos, reduciendo los efectos de variación somaclonal que se pueden presentar en el material vegetal y conservando fidedignamente la estructura genética de las accesiones.

\section{Literatura citada}

1. Aguirre, C., Mancipe, C. \& Bonilla, M. (2015). Conservación in vitro: una perspectiva para el manejo de los recursos fitogenéticos. I Simposio Colombiano de Recursos Fitogenéticos Neotropicales-VIII Congreso Colombiano de Botánica. 102 pp.

2. Azcon-Bieto J. \&, Talon M. (2008). Fundamentos de fisiología vegetal. 2da edición. Mc Graw-Hill. New York, NY. 656 pp.

3. Barnicoat, H., Cripps, R., Kendon J. \& Sarasan, V. (2011). Conservation in vitro of rare and threatened ferns-case studies of biodiversity hotspot and island species. Cell. Dev.Biol. Plant. 47: 37-45.

4. Bonilla, M., Pachón, J., Quiñonez, L. (2012). Establecimiento de banco de germoplasma de Dioscorea spp. de la Orinoquía Colombiana: potencial uso en la industria. V Congreso Internacional de Plantas Medicinales: productos naturales y biodiversidad, Universidad Nacional de Colombia, Sede Palmira. 50pp.

5. Bonilla, M. \& Hernández, O. (2012). Propagación in vitro de ñame (Dioscorea spp.): una perspectiva en la producción masiva de plantas y conservación de germoplasma. Revista Agronomía. 20 (2): 65-76.

6. Bonilla, M. \& Caetano, C. (2013). Inventario y valoración de la flora utilizada por la vereda Santa Teresa, Palmira (Valle del Cauca). Revista de Investigación Agraria y Ambiental.4 (1):89 -99.

7. Biodiversity international. (2011). Is cryopreservation a viable method for longterm conservation of coffee biodiversity? Recuperado de: www.biodiversityinernational.org

8. Bunnag, S. \& Khonkayan, S. (2010). Long-term preservation of Dendrobium chrysotoxum Lindl. using encapsulation method. Bioflux. 2 (2): 45-50.

9. Calva, G. \& Pérez, J. (2005). Cultivo de células y tejidos vegetales: la fuente de alimentos del futuro. Revista Digital Universitaria. 6(11): 1-16.

10. Daskalova, E., Dontcheva, S., Yahoubian, G., Minkov, I. \& Toneva V. (2011). A strategy for conservation and investigation of the protected resurrection plant Haberlea rhodopensis Friv. BioRisk. 6: 41-60.

11. Divakaran, M, Nirmal, K. \& Peter K. (2006). Conservation of Vanilla species, in vitro. Scientia Horticulturae .110: 175-180.

12. Engelmann, F. (2011). Use of biotechnologies for the conservation of plant biodiversity. In vitro Cell. Dev. BiolPlant. 47:5-16.

13. FAO. (2010). Tratado internacional sobre los recursos fitogenéticos para alimentación y la agricultura. Recuperado de: ftp://ftp.fao.org/docrep/fao/011/i0510s/i0510s.pdf 
14. Faisal, N., Ahmad N. \& Anis, M. (2006). In vitro plant regeneration from alginate-encapsulated microcutting of Rauvolfia tetraphylla L. American-Eurosian J. Agric y Environ. Sci. 1 (1): 01-06.

15. Gagliardi, R., Hanai, L., Pacheco, G., Oliveira, C., Carneiro, L., Montenegro, J., Mansur, E. \& Carneiro, M. (2007). Assessment of Genetic Stability Among In vitro Plants of Arachis retusa. Using RAPD and AFLP Markers for germplasm preservation. JIPB. 49 (3): 307-312.

16. García, L., Feria, M. \& Acosta, K. (2007). Aspectos básicos de la conservación in vitro de germoplasma vegetal. Biotecnolog ía Vegetal. 7 (2): 67 - 79.

17. Germana, M., Micheli, M., Pulcini, L. \& Standardi A. (2007). Perspectives of the encapsulation technology in the nursery activity of Citrus. Caryologia. 60 (1-2): 192-195.

18. González, M. \& Martín, C. (2011). In vitro Preservation of Spanish Biodiversity. In vitro Cell.Dev.Biol.Plant. 47: 46-54.

19. Guo, B., Stiles, A. \& Liu, C. (2013). Low-temperature preincubation enhances survival and regeneration of cryopreserved Saussurea involucrate Callus. In vitro Cell.Dev.Biol.-Plant. 49: 320-325.

20. Hidalgo, R. \& Vallejo, F. (2014). Base para el estudio de los recursos genético de especies cultivadas. Universidad Nacional de Colombia, Sede Palmira. 284pp.

21. Iriondo, J. (2011). Conservación de germoplasma de especies raras y amenazadas. Investigación AgrariaProducción y protección vegetal. 16 (1): 5-24.

22. Jung, Y., Sok, Y., Gi, A., Jong, W., Ji, H. \& Haeng, $H$. (2012). Improvement of the Doplet-vitrification Method for the Cryopreservation of Cultivated Potato Shoot Tips. Kor. J. Breed. Sci. 44(2): 94-99.

23. Kavand, S., Kermani, M., Haghnazari, A., Khosravi, P., \& Reza, M. (2011). Micropropagation and mediumterm conservation of Rosa Pulverulenta. Maringá. 33 (2):297-301.

24. Kaviani, B., Darabi A. \& Roudposhti, V. (2012). In vitro Conservation of Genetic Resources of Tea (Camellia sinenesis L. cv.100) using Storage of Germplasms (Embryonic Axes and Shoot Tips) in Cryopreservation Conditions. Annals of Biological Research. 3 (7):3541-3546

25. Kokowicz, F., Aguiar, T., Simões, T., Benson, E. \& Viana, A. (2011). In vitro and cryogenic preservation of plant biodiversity in Brazil. In vitro Cell.Dev.Biol.-Plant. 47:82-98.

26. Krishnan, P., Decruse, S. \& Radha, R. (2011). Conservation of medicinal plants of Western Ghats, India and its sustainable utilization through in vitro technology. In vitro Cell.Dev.Biol.-Plant. 47:110-122.

27. Lima, A., Sampaio, M., Matos, B., Resende, S., Cortizo, M. \& Ferreira, J. (2011). Agentes osmóticos e temperature na conservação in vitro de sempre-viva. Ciencia Rural. 41 (8): 1354-1361.

28. Maneerattanarungroj, P., Bunnag, S. \& Monthatong, M. (2007). In vitro conservation of Cleisostoma areitinum (Rchb.f.) Garay, rare Thai Orchid species an encapsulation-dehydration method. Asian Journal of Plant Sciences. 6 (8): 1235-1240.
29. Matos, E., Marcano, M., Azócar, C. \& Mora, A. (2015). Establecimiento y multiplicación in vitro de cinco cultivares de Apio (Arracacia xanthorriza Bancroft) colectados en Venezuela. Bioagro. 27 (2): 121-130.

30. Martín, I. (2006). Conservación de recursos fitogenético. Recuperado de:http://www.esporus.org/recursos/ articles/agrobiodiversitat/conservacion_rec_fi tog_ isaura_martin.pdf

31. Mohan, S. (2011). Prospects of in vitro conservation of date palm genetic diversity for sustainable production. Emir. J. Food Agric. 23 (2): 110-119

32. Normah, M., Choo, W., Yap, L. \& Mohamed, Z. (2011). In vitro conservation ofMalaysian biodiversity-achievements, challenges and future directions. In vitro Cell. Dev. Biol-Plant. 47:26-36.

33. Ozudogru, E., Kirdok, E., Kaya, E., Capuana, M., De Carlo, A. \& Engelmann, F. (2011). Medium-term conservation of redwood (Sequoia sempervirens (D. Don.) Endl.) in vitro shoot cultures and encapsulated buds. Scientia Horticulturae. 127: 431-435.

34. Pence, V. (2011). Evaluating costs for the in vitro propagation and preservation of endangered plants. In vitro Cell. Dev. Biol-Plant.47:176-187.

35. Pimda, W. \& Bunnag, S. (2010). Cryopreservation of Dendrobium heterocarpum Lindl. Via encapsulationdehydration method. Bioflux. 2 (1): 7-14.

36. Radovet, D. \& Cahita, D. (2012). In vitro conservation of some potato genotypes to improve the ecoeconomic impact of plant biotechnology. Vasile Goldis. 22 (4): 511-518.

37. Ramírez, H., Guevara, M. \& Escobar, R. (2012). Cultivo de tejidos vegetales, conceptos y prácticas. Universidad Nacional de Colombia, Sede Palmira. Editorial Feriva S.A, Cali, Colombia. 227 pp.

38. Raveendran, R., Kavitha, M., Thilaga, S. \& D. Ganesh. (2012). Conservation and in vitro multiplication of highly endangered Indian traditional medicinal plant (Morinda reticulata Gamble) through nodal explants. Plant Knowledge Journal. 1(2)46-51.

39. Reed, B., Sarasan, V., Kane, M., Bunn, E. \& Pence, V. (2011). Biodiversity conservation and conservation biotechnology tools. In vitro Cell. Develoment Biol-Plant. 47:1-4.

40. Rivera, A., Valbuena, R., Hidalgo R. \& Moreno, J. (2008). Crioconservación de yemas de microtubérculos de papa Solanum tuberosum ssp. andigena mediante desecado de tejidos. Revista Corpoica. 9(2): 37-44.

41. Roca, W., Arias, D. \& Chávez, R. (1991). Métodos de conservación in vitro del germoplasma. In: Roca, W. \& Mroginski, L., Cultivo de tejidos en la agricultura. CIAT.1-969 pp.

42. Rowntree, J., Pressel, S., Ramsay, M., Sabovljevic A. \& Sabovljevic, M. (2011). In vitro conservation of European bryophytes. In vitro Cell. Dev. Biol-Plant.47:55-64.

43. Sans, F. (2007). La diversidad de los agroecosistemas. Ecosistemas. 16 (1): 44-49.

44. Sánchez, N. \& Jiménez, V. (2010). Técnicas de conservación in vitro para el establecimiento de bancos de germoplasma en cultivos tropicales. Agronomía Mesoamericana. 21 (1): 193-205. 
45. Scocchi, A., Faloci, M., Medina, R., Olmos S. \& Mroginski, L. (2004). Plant recovery of cryopreserved apical meristem-tips of Melia azedarach L. using encapsulation/dehydration and assessment of their genetic stability. Euphytica. 135: 29-38.

46. Scott, G., Rosegrant, M. \& Ringler, C. (2000). Raíces y tubérculos para el siglo 21: Tendencias, proyecciones y opciones de política. Instituto Internacional de Investigación sobre Políticas Alimentarias. 64pp.

47. Shaheen, A. \& Shahzad, A. 2015. Nutrient encapsulation of nodal segments of an endangered whitecedar for studies of regrowth, short term conservation and ethylene inhibitors influenced ex vitro rooting. Industrial Crops and Products. 69: 204-211.

48. Shekhawat, M., Kannan, N., Manokari, M. \& Ravindran, C. (2015). In vitro regeneration of shoots and ex vitro rooting of an important medicinal plant Passiflora foetida L. through nodal segment cultures. Journal of Genetic Engineering and Biotechnology. doi:10.1016/j. jgeb.2015.08.002

49. Shukla, M., Jones, A., Sullivan, J. \& Liu, C. (2012). In vitro conservation of American elm (UImus americana): potential role of auxin metabolism in sustained plant proliferation. Can. J. For. Res. 42: 686-697.

50. Silva, J., Espinosa, A., Acosta, L., González, O., Licea, R. \& Meneses, S. (2001). Resultados y perspectivas de la conservación in vitro de recursos fitogenéticos en la región oriental de Cuba. Cuadernos de Biodiversidad. 7 (1): 4-6.

51. Simpson, M. 2010. Plant systematics. Academic Press. Second Edition. 740pp.

52. Tavazza, R., Alonso, R., Papacchioli, V. \& Pagnotta, L. (2015). A validated slow-growth in vitro conservation protocol for globe artichoke germplasm: A cost-effective tool to preserve from wild to elite genotypes. Scientia Horticulturae. doi:10.1016/j.scienta.2015.09.024

\section{Conflicto de Intereses}

Los autores declaran no tener ningún conflicto de intereses 
UNAP 